\title{
Beam-Riding Analysis of a Parabolic Laser-thermal Thruster
}

\author{
Stefan Scharring ${ }^{*}$, Hans-Albert Eckel ${ }^{*}$ and Hans-Peter Röser ${ }^{\dagger}$ \\ *Institute of Technical Physics, German Aerospace Center, \\ D-70569 Stuttgart, Pfaffenwaldring 38-40, Germany \\ $\dagger$ Institute of Space Systems, University of Stuttgart, \\ D-70569 Stuttgart, Pfaffenwaldring 31, Germany
}

\begin{abstract}
Flight experiments with laser-propelled vehicles (lightcrafts) are often performed by wire-guidance or with spin-stabilization. Nevertheless, the specific geometry of the lightcraft's optics and nozzle may provide for inherent beam-riding properties. These features are experimentally investigated in a hovering experiment at a small free flight test range with an electron-beam sustained pulsed $\mathrm{CO}_{2}$ high energy laser. Laser bursts are adapted with a real-time control to lightcraft mass and impulse coupling for ascent and hovering in a quasi equilibrium of forces. The flight dynamics is analyzed with respect to the impulse coupling field vs. attitude, given by the lightcraft's offset and its inclination angle against the beam propagation axis, which are derived from the 3Dreconstruction of the flight trajectory from highspeed recordings. The limitations of the experimental parameters' reproducibility and its impact on flight stability are explored in terms of Julia sets. Solution statements for dynamic stabilization loops are presented and discussed.
\end{abstract}

Keywords: Laser propulsion, Free flight experiments, Lightcraft, Repetition rate, Impulse coupling coefficient, High speed analysis, $\mathrm{CO}_{2}$ laser, Stabilization, Beam-Riding

PACS: 05.45.-a, 05.45.Gg, 42.62.-b, 45.40.-f, 52.50.Jm, 52.77.-j

\section{INTRODUCTION}

Energy supply by a remote source plays a key role in beamed energy propulsion. Hence, the beam-riding properties of a laser-driven device (lightcraft) are of major importance for the maturity of this innovative propulsion technology. Stabilization techniques are widely used in rocketry and spin-stablization contributed to the success of the lightcraft's world record flight of Myrabo [1]. Nevertheless, the knowledge of the inherent beamriding characteristics of a laser-propelled device is important for the layout of appropriate, cost-effective and light-weight auxiliary stabilization techniques. Beam-riding properties have been characterized using the Angular Impulse Measuring Device (AIMD) of Myrabo's group: Lateral and angular momentum were measured with respect to the offset from beam center and an oscillatory lateral motion of spin-stabilized flights was reported [2-5].

Beam-riding experiments at DLR Stuttgart with a parabolic laser lightcraft have been reported in $[6,7]$. Since the parabolic lightcraft was investigated without spinstabilization, the angular motion of the vehicle is of special interest additionally to the lateral motion. A detailed model of intensity distribution and resulting impulse components is given in [8] together with corresponding flight performance predictions based on Julia sets. 
In the following, the results of a beam-riding experiment are presented that focuses on the lateral and angular motion. We managed to create a dynamic equilibrium of propulsive laser power and gravity establishing a nearly stable hovering state for a short time. Launch position and laser burst adjustment were varied as critical parameters for beam-riding stability.

\section{THEORY}

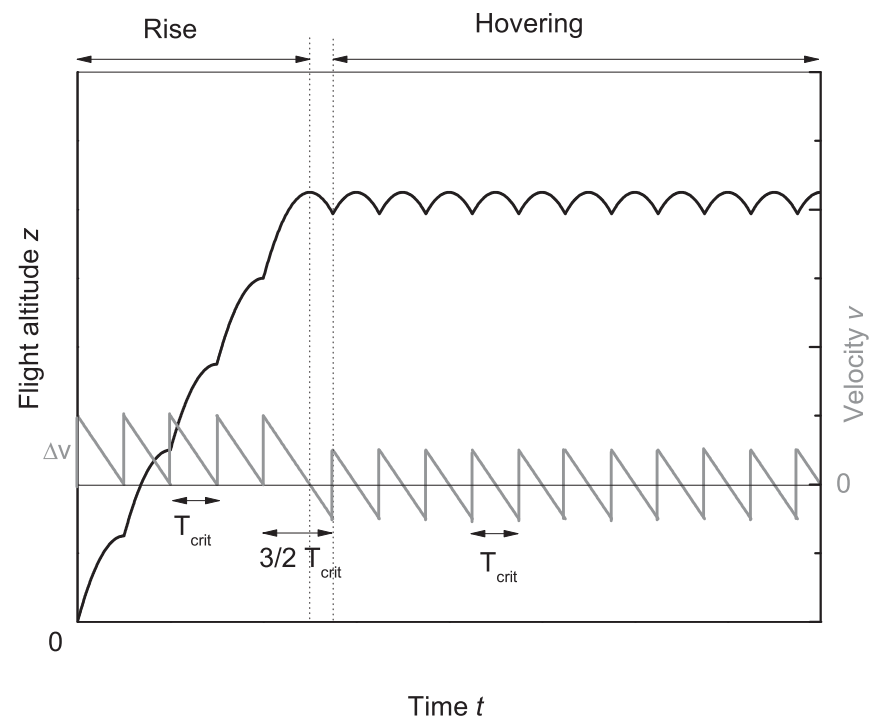

FIGURE 1. Scheme of the temporal course of flight altitude $z$ and vertical velocity $v$ in a hovering experiment.

The basic idea of a hovering experiment in pulsed laser propulsion is given in Figure 1: The gravitational force of the lightcraft is compensated if the condition

$$
c_{m}\left(E_{L}\right)=\frac{m_{L C} \cdot \Delta v}{E_{L}}=\frac{m_{L C} \cdot g}{E_{L} \cdot f_{r e p}}
$$

is met, where $c_{m}$ is the coupling coefficient, $E_{L}$ the laser pulse energy, $m_{L C}$ the lightcraft's mass, $\Delta v$ the lightcraft's velocity increment by the laser pulse, $g$ the gravitational acceleration and $f_{\text {rep }}$ the laser pulse repetition rate. Then, the critical laser pulse period is defined as $T_{c r i t}=f_{\text {rep }}^{-1}$. In this case, the vehicle rises during the first phase of the experiment in average without any residual acceleration. Each laser pulse is fired when the vehicle's velocity equals zero. After the lightcraft's rise to its hovering altitude, the laser burst is interrupted for $0.5 \cdot T_{\text {crit }}$ and afterwards restarted again. Now, the hovering phase commences with laser pulses at each time when $v=-0.5 \cdot \Delta v$, where $\Delta v$ is the velocity increment of the vehicle induced by a laser pulse of the energy $E_{L}$. Again, in average no residual acceleration occurs. Hence, with a perfectly reliable laser system this state should theoretically be stable, if the vertical motion was not affected by angular and 
lateral impulse components. For analysis of these impulse components, we re-define $c_{m}$ as a vectorial parameter $\vec{c}_{m}$, where the lateral components $c_{m, x}$ and $c_{m, y}$ represent the lateral momentum transfer, i.e. vertical to the beam propagation axis, and $c_{m . z}$ is the component in direction of beam propagation, commonly known as $c_{m}$. In a similar way, the rotational impulse coupling coefficient $\vec{c}_{L}$ can be defined as

$$
\vec{c}_{L}=\frac{J \cdot \vec{\omega}}{E_{L}}
$$

where $J$ denotes the momentum of inertia with respect to the corresponding axis and $\omega_{i}$ represents the angular velocity of the lightcraft's inclination $\vartheta_{i}$ in the projection of the $i-z$ plane. In the following, a counter-clockwise inclination is positive.

\section{EXPERIMENTAL}

\section{Lightcraft}

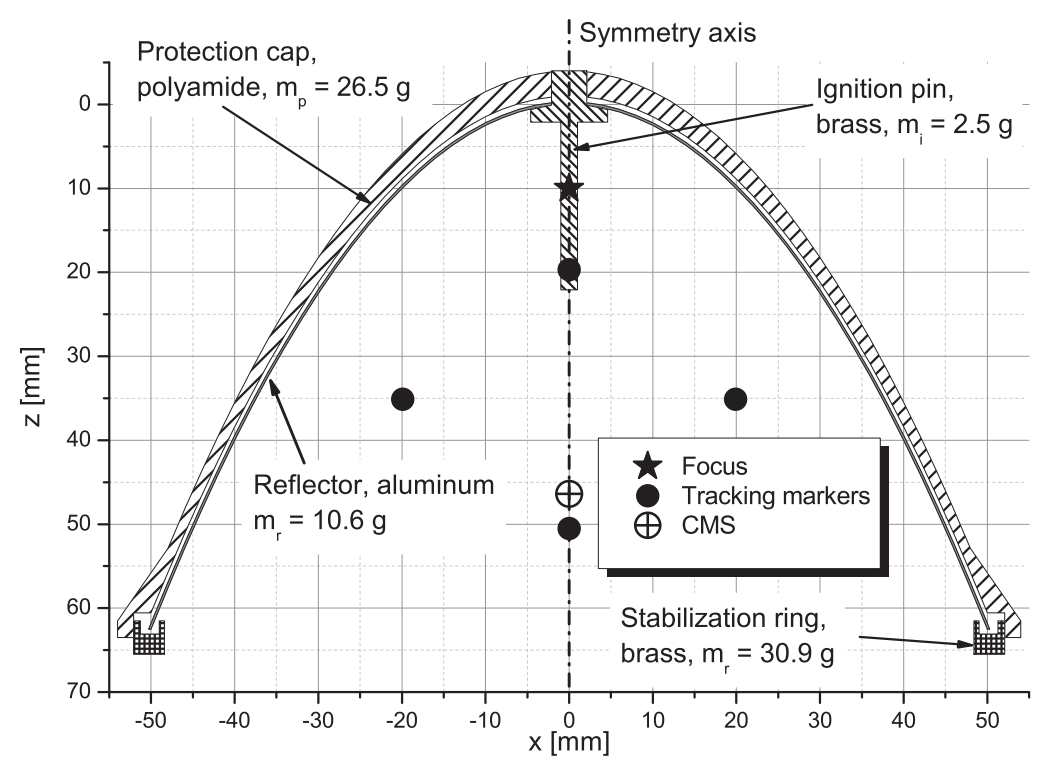

FIGURE 2. Cross-section of the lightcraft with parabolic reflector, protection cap, ignition pin and stabilization ring. For experiments $\mathrm{B}$ and $\mathrm{C}$, a modified hull with $m_{p}=24.8 \mathrm{~g}$ was employed where the height was adapted to the stabilization ring. The reflector's apex is located in the axes' origin.

A cross-section of the lightcraft is shown in Figure 2. The parabolic mirror made of aluminum by metal pressing serves as thrust chamber. It exhibits a diameter of $100 \mathrm{~mm}$, $62.5 \mathrm{~mm}$ height and a focal length of $10 \mathrm{~mm}$. For shock protection at dropdown after the flight, a cap made of polyamide by laser-sintering is attached to the parabolic reflector. Preliminary experiments have shown a poor hovering stability for a regular lightcraft. Hence, for hovering experiments, a stabilization ring made of brass was attached to the nozzle exit. The moment of inertia and the location of the center of mass of the craft's 
components was derived from model calculations with Solid Edge. With the stabilization ring, the CMS was lowered from $z=33.0 \mathrm{~mm}$ down to $z=46.4 \mathrm{~mm}$ and the momentum of inertia was enlarged from $\approx 910 \mathrm{~g} \cdot \mathrm{cm}^{2}$ to $\approx 1470 \mathrm{~g} \cdot \mathrm{cm}^{2}$, diminishing the magnitude of the angular motion.

\section{Laser}

An electron-beam sustained $\mathrm{CO}_{2}$ high energy laser was used as a power source for the propulsion of the laser lightcraft. The laser was operated in a stable resonator configuration with pulse energies in the range of $E_{L} \approx 25-175 \mathrm{~J}$ and pulse lengths of $\tau=0.29-0.37 \mu s$ (spike) and $\tau=7.2-10.0 \mu s$ (tail, $88-96 \%$ of $E_{L}$ ). In repetitive operation, however, the pulse energy was limited, e.g. at $f_{\text {rep }}=20 \mathrm{~Hz}$ to $\approx 110 \mathrm{~J}$. With higher pulse energies, arc discharges in the laser cavity occured relatively often in spite of the recirculation of the laser gas. This impaired performance is mainly due to aging processes in the resonator cavity.

The laser beam propagation in the range of 0.9 to $3.5 \mathrm{~m}$ was analyzed with thermal paper yielding a beam quality of $M_{x}^{2}=81$ and $M_{y}^{2}=71$, resp., cf. [9]. At the output coupler, the laser beam exhibits a diameter of $d_{x}=80 \mathrm{~mm}$ and $d_{y}=79 \mathrm{~mm}$, resp., on its principal axes. The laser burst was controled by an FPGA chip on a cRIO chassis (\# 9072 by National Instruments). A script was written for a digital I/O module (\# 9401 by National Instruments) providing for a burst sequence with selectable pulse periods according to Figure 1. The trigger pulse width was set to $5 \mathrm{~ms}$, the inhibit time after firing before reloading the laser's pulse forming network was $10 \mathrm{~ms}$, cf. [10].

In first tests, the real-time control was connected to the external input of the main laser control unit. However, reproducibility of the laser bursts was severely impaired by electromagnetic noise from the frequency converter of the axial fans and the laser discharge itself and was not reached until the cRIO output was connected with a former remote control of the laser which was joined directly with the circuit board of the main laser control by optical waveguides.

\section{Measurement techniques}

In the flight experiment, cf. Figure 3, the lightcraft is placed on a launchpad consisting of 3 steel rods in a $120^{\circ}$ configuration inside a large optical mount. The launchpad is placed above a planar copper mirror (\# 1) that bends the laser beam into the vertical direction. For alignment purposes, a HeNe laser is coupled through a small aperture at the rear mirror of the $\mathrm{CO}_{2}$ laser coaxially through the laser cavity. Its diameter is reduced with a pinhole which served as screen to control the inclination of the launchpad by means of backreflection. Therefore, the lightcraft is replaced by a planar mirror. Precise alignment is given when the HeNe beam is reflected back through the pinhole. Afterwards, for a precise positioning of the lightcraft, the planar mirror was removed and the lightcraft's ignition pin was adjusted to the HeNe spot. 


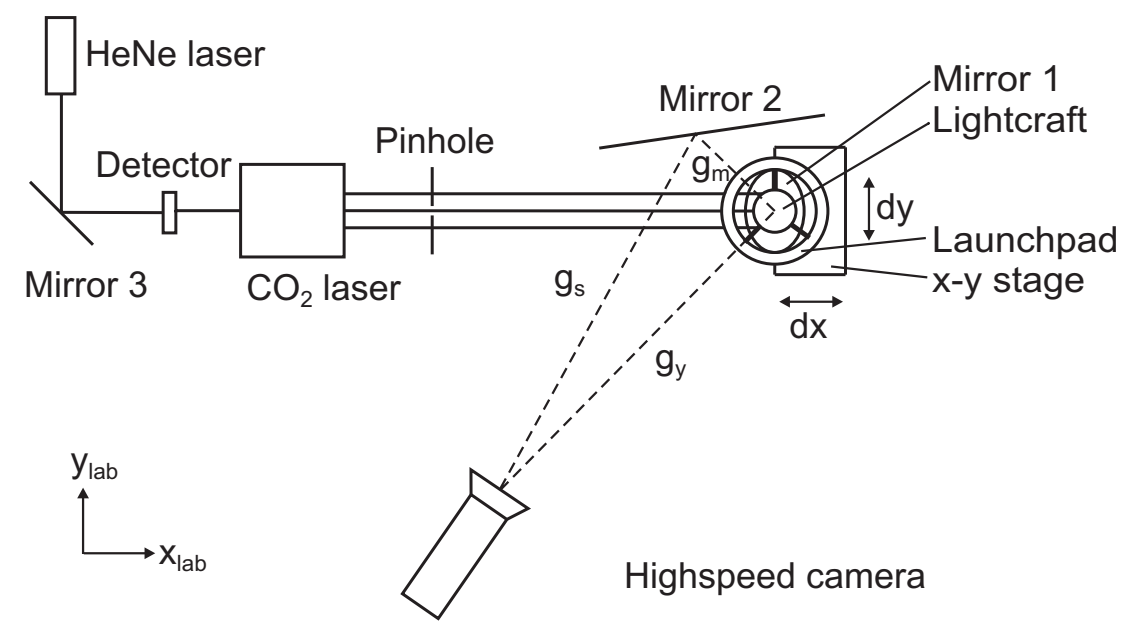

FIGURE 3. Scheme of the setup for hovering experiments

The flight path was monitored by a highspeed camera, MotionScope M3 by Redlake, with a framerate of 500 fps. Mirror \# 2 enabled for a stereoscopic view of the flight from two directions in a $90^{\circ}$ angle. These directions correspond to the principal axes of the laser beam. Since the distance between camera and objects differs between both images $\left(g_{x}=g_{s}+g_{m}=1.20 m, g_{y}=0.91 \mathrm{~m}\right)$, aperture settings were adjusted both to the depth of focus and the setup illumination $(\kappa=5.6, f=35 \mathrm{~mm})$. The flight path was illuminated with a LED array (Constellation 19-LED light by Imaging Solutions) that was synchronized with the highspeed camera. In later experiments, synchronization failed due to strong electromagnetic noise, cf. the previous section. Offline tracking of the markers on the protection cap of the lightcraft allows for reconstruction of the flight trajectory, as described in detail in [6]. The frames of the flight path were calibrated with two grids in each direction.

After alignment, the pinhole was removed and a pyrodetector (PE50BB-V2 by Ophir Optronics) was placed in front of the rear aperture of the $\mathrm{CO}_{2}$ laser for detection of a small fraction $(1 /(711 \pm 71))$ of the entire laser pulse energy. The detector was attached to a control unit (Laserstar Dual Channel, later: Pulsar-2 by Ophir), the latter one allowed for monitoring of the pulse times. The laser pulses were monitored as well at the pulse forming network of the laser's main discharge with an oscilloscope (LT 342 Waverunner by LeCroy) in order to detect laser malfuncion, e.g. arc discharges, and for camera triggering.

\section{RESULTS AND DISCUSSION}

Three experimental campaigns were performed with a predefined real-time controlled laser burst, cf. Tab. 1. While most of the laser bursts were synchronized precisely with the real-time control, cf. data for the cycle-to-cycle jitter $J_{C C}=\max \left|T_{n+1}-T_{n}\right|$, the reproducibility of the flight trajectories was mainly impaired by the large pulse-to-pulse jitter $J_{P P}=\max \left|E_{L, n+1}-E_{L, n}\right|$ of the laser pulse energy. However, some laser bursts 
TABLE 1. Parameters of hovering experiments with predefined laser burst

\begin{tabular}{|c|c|c|c|}
\hline & \multicolumn{3}{|c|}{ Datasets } \\
\hline & $\mathbf{A}$ & B & $\mathbf{C}$ \\
\hline Flight ID & $1734-1777$ & $1825-1853$ & $1905-1920$ \\
\hline \multicolumn{4}{|l|}{ Laser } \\
\hline$U_{P F N}[k V]$ & 32.5 & 30 & 32.5 \\
\hline$E_{L, \text { Burst }}[J]$ & $109.5 \pm 3.5$ & $84.1 \pm 4.1$ & $111.3 \pm 1.9$ \\
\hline$J_{P P}[J]$ & $9.4 \pm 2.4$ & $8.8 \pm 3.1$ & $10.3 \pm 3.0$ \\
\hline$N_{\text {misfire }}$ & 9 & 4 & 1 \\
\hline \multicolumn{4}{|l|}{ Lightcraft } \\
\hline$m_{L C}[g]$ & 70.47 & 68.73 & 68.73 \\
\hline$J_{L C}\left[\mathrm{~g} \cdot \mathrm{cm}^{2}\right]$ & $1467 \pm 73$ & $1410 \pm 70$ & $1410 \pm 70$ \\
\hline$\Delta v^{(1)}[\mathrm{mm} / \mathrm{s}]$ & $489 \pm 28$ & $263 \pm 26$ & $407 \pm 17$ \\
\hline$E_{L}^{(1)}[J]$ & $112 \pm 13$ & $85 \pm 10$ & $109 \pm 13$ \\
\hline$c_{m}^{(1)}[N / M W]$ & $307 \pm 40$ & $213 \pm 33$ & $257 \pm 32$ \\
\hline$T_{\text {crit }}[\mathrm{ms}]$ & $49.8 \pm 2.9$ & $26.8 \pm 2.7$ & $41.5 \pm 1.7$ \\
\hline \multicolumn{4}{|c|}{ Real-time control } \\
\hline$T_{\exp }[m s]$ & 44 & 24 & 38 \\
\hline$N_{T \text { misaligned }}$ & 4 & 11 & 4 \\
\hline$J_{C C}[\mu s]$ & $8 \pm 19$ & $297 \pm 132$ & $42 \pm 80$ \\
\hline$N_{\text {rise }}[$ periods $]$ & 5 & 4 to 5 & 5 \\
\hline$P_{a v}[k W]$ & $2.5 \pm 0.3$ & $3.5 \pm 0.4$ & $2.9 \pm 0.3$ \\
\hline \multicolumn{4}{|l|}{ Launch } \\
\hline$v_{l}[m / s]$ & $0.30 \pm 0.02$ & $0.16 \pm 0.02$ & $0.24 \pm 0.01$ \\
\hline$a_{\text {res }}[G]$ & $-0.042 \pm 0.020$ & $-0.053 \pm 0.066$ & $-0.038 \pm 0.017$ \\
\hline \multicolumn{4}{|l|}{ Hovering } \\
\hline$N_{\text {flights }}$ & 31 & 14 & 9 \\
\hline$N_{\text {hov }}[$ periods $]$ & 1 to 8 & 0 to 15 & 0 to 5 \\
\hline$t_{\text {hov }}[m s]$ & $153 \pm 93$ & $103 \pm 111$ & $127 \pm 54$ \\
\hline$z_{\text {hov }}[\mathrm{mm}]$ & $57 \pm 8$ & $19 \pm 12$ & $40 \pm 5$ \\
\hline
\end{tabular}

( $\left.N_{\text {misfire }}\right)$ exhibited missing or temporally shifted laser pulses and in general, with higher repetition rates, $J_{C C}$ increased significantly.

Two different voltage settings $U_{P F N}$ at the pulse forming network of the laser's main discharge were selected yielding laser pulse energies $E_{L} \approx 85 \mathrm{~J}$ and $\approx 110 \mathrm{~J}$. The theoretical repetition period $T_{\text {crit }}$, cf. Fig. 1, can be derived according to Eq. 1 from the velocity increment $\Delta v^{(1)}$ at the first laser pulse. Since lateral offset and angular tilt dimished $c_{m, z}$ during the 5 pulses of the lightcraft's ascent, in the first test flights $\left(N_{T \text { misaligned }}\right)$ the repetition period was too long, and a shorter laser period, $T_{\text {exp }}$, had to be chosen, yielding an average laser power $P_{a v}$ that compensated the lightcraft's gravitational force in a hovering state of $N_{\text {hov }}$ periods and $t_{\text {hov }}$ duration, resp. 


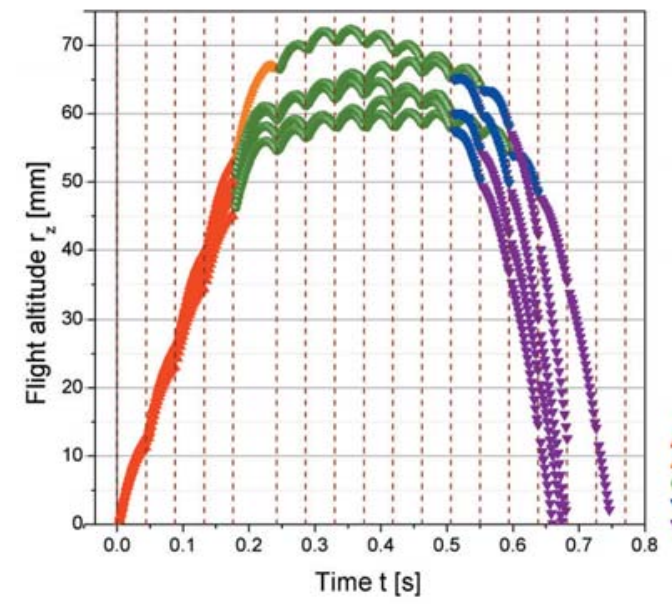

(a)

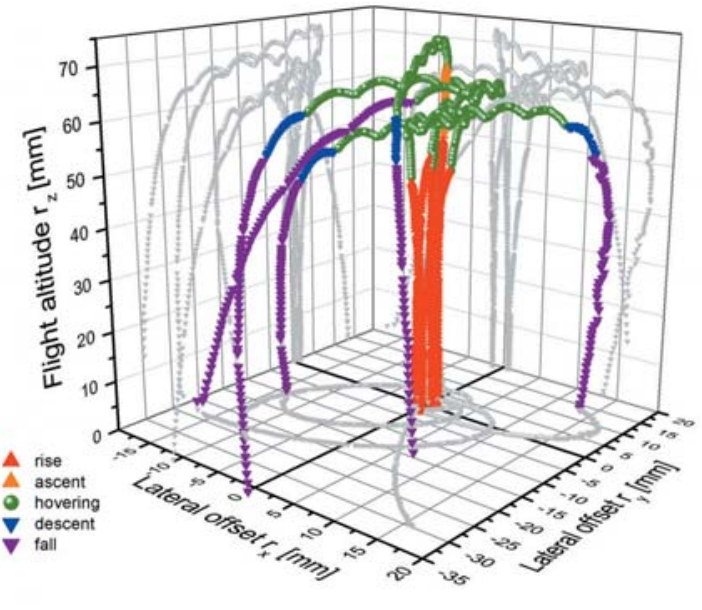

(b)

FIGURE 4. Altitude vs. time graph (a) and corresponding trajectories (b) for selected hovering flight experiments (ID 1741, 1742, 1743, 1749, and 1751) from dataset A. The dashed lines indicate the laser pulses.

\section{Vertical motion}

Figure 4 shows the temporal course of the flight altitude for selected flights with a relatively long hovering state. For characterization of the flight period between the $n^{\text {th }}$ and the $n+1^{\text {st }}$ pulse, we define an index $f_{i}$ with

$$
f_{i}=\frac{v_{n}^{(+)}}{v_{n}^{(+)}-v_{n+1}^{(-)}} \approx \frac{v_{n}^{(+)}}{g \cdot T n}
$$

where $v_{n}^{(+)}$denotes the vertical velocity $v_{z}$ instantaneously after the $n^{\text {th }}$ laser pulse and $v_{n+1}^{(-)}$refers to the $v_{z}$ directly before the subsequent pulse. The velocities were derived from datafits of the flight trajectories. We define a hovering period for a flight segment where $0.25<f_{i}<0.75$, i.e., if the main time the upward motion is compensated by the motion downwards. Rise and fall of the lightcraft can be clearly defined according to $f_{i} \geq 1$ and $f_{i} \leq 0$, resp. The remaining cases are transition states, denoted as ascent and descent, resp.

In first phase of the flight, the lightcraft rises in a state where gravitational force and laser power nearly compensate each other. In average, the launch velocity $v_{l}$ is therefore nearly constant, and the residual acceleration $a_{\text {res }}$ is rather low, cf. Tab. 1 . The hovering altitude $z_{\text {hov }}$, i.e., averaged over the periods with $0.25<f_{i}<0.75$, amounts only several centimeters. In spite of the continuous firing of the laser up to $\approx 1 s$, the lightcraft starts falling down at $t=0.5 \mathrm{~s}$ at the latest. One reason for this behavior can be deduced from the flight trajectories as shown in Fig. 4 (b). During the hovering phase, the lightcraft starts drifting away from the beam center and $c_{m}$ obviously decreases.

However, the ground trajectories show an arc-shaped lateral motion indicating back- 


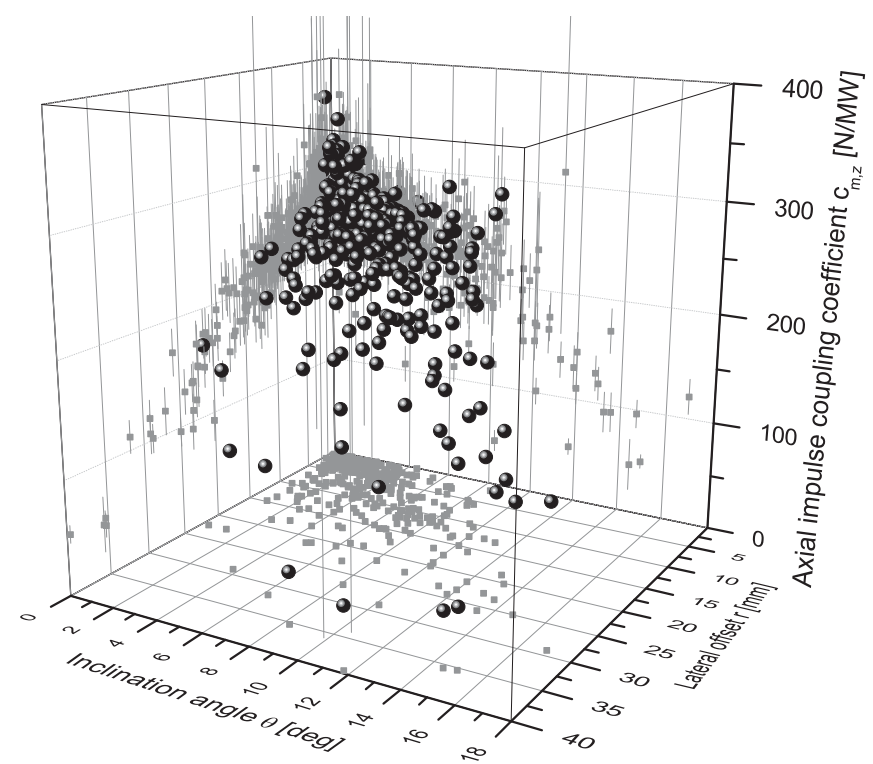

FIGURE 5. Axial impulse coupling, dataset A.

TABLE 2. Fitting results for axial momentum. $N_{p}$ is the number of datapoints.

\begin{tabular}{lrrrrr}
\hline Dataset & $\begin{array}{r}c_{z} \\
N \cdot M W^{-1}\end{array}$ & $\begin{array}{r}\sigma_{r} \\
m m\end{array}$ & $\begin{array}{r}\sigma_{\vartheta} \\
d e g\end{array}$ & $N_{p}$ & $\bar{R}^{2}$ \\
\hline $\mathrm{A}$ & $273.9 \pm 1.5$ & $26.4 \pm 0.8$ & $28.4 \pm 2.8$ & 383 & 0.80 \\
$\mathrm{~B}$ & $185.0 \pm 3.8$ & $38.3 \pm 6.4$ & $33.7 \pm 9.7$ & 187 & 0.12 \\
$\mathrm{C}$ & $229.0 \pm 2.7$ & $25.6 \pm 1.0$ & n.d. & 106 & 0.84 \\
Model $^{\dagger}$ & n.d. $^{* *}$ & $36.8 \pm 0.1$ & $3.14 \pm 0.01$ & 8957 & 0.96 \\
\hline
\end{tabular}

* In this dataset, no pronounced dependency on $\vartheta$ was found.

$\dagger$ data from raytracing, cf. [8]

** arbitrary units

driving momentum components, as reported previously in [6] and [2]. Hence,the dependency of $c_{m, z}$ from lateral offset and inclination angle was analyzed for each laser pulse of the hovering flights inducing momentum by a datafit of

$$
c_{m, z} \approx \frac{c_{z}}{\left[1+\left(\frac{r}{\sigma_{r}}\right)^{2}\right]\left[1+\left(\frac{\vartheta}{\sigma_{\vartheta}}\right)^{2}\right]}
$$

cf. Fig. 5 and Tab. 2. 


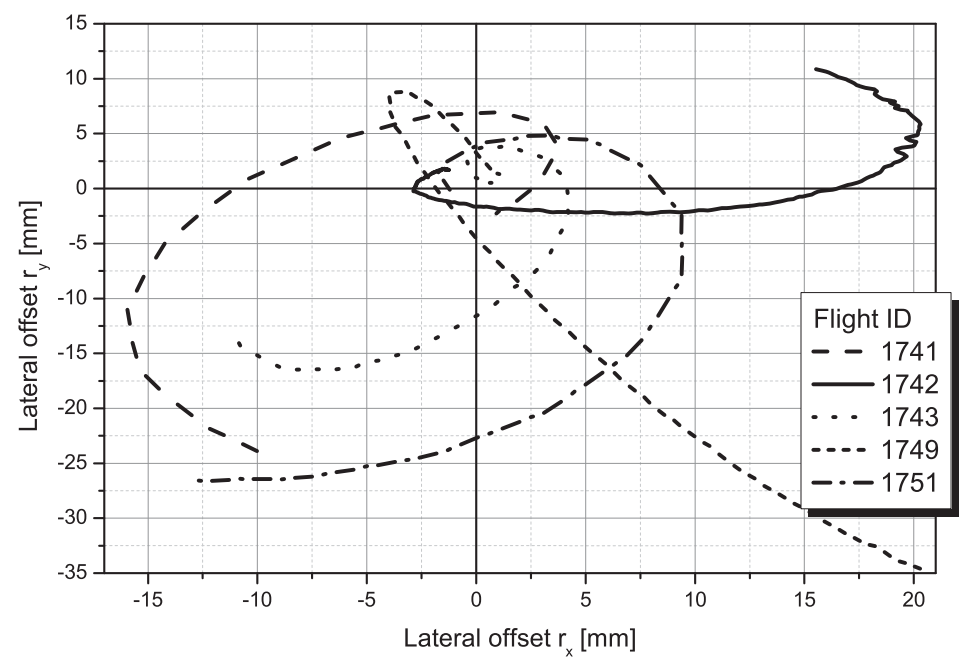

(a)

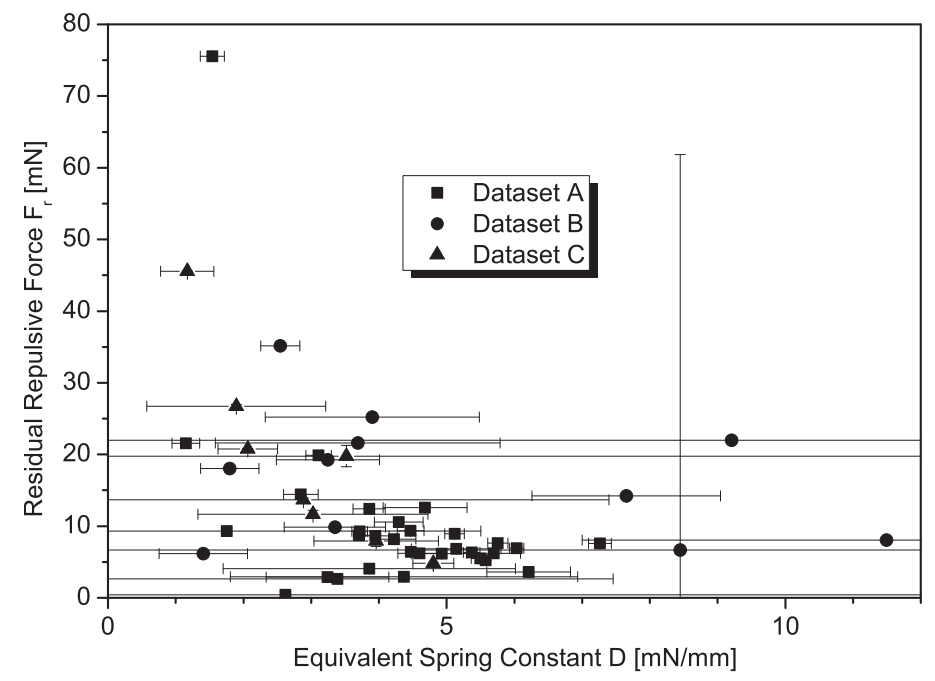

(b)

FIGURE 6. Lateral motion: The spiralform ground trajectories (a) can be deduced from (b) an equivalent spring constant $D$ and a residual repulsive force $F_{r}$.

\section{Lateral motion}

During the hovering period, the average lateral offset of the lightcraft ranged from $6.2 \pm 5.4 \mathrm{~mm}$ (dataset B) to $9.6 \pm 4.2 \mathrm{~mm}$ (dataset C). The lateral velocity was in the range of $64 \pm 59 \mathrm{~mm} / \mathrm{s}(\mathrm{B})$ to $107 \pm 29 \mathrm{~mm} / \mathrm{s}(\mathrm{C})$, however, the ground trajectories of the flights in the $x-y$ plane exhibit a characteristic spiralform shape, cf. Fig. 6 (a). Hence, 
the temporal courses of $r_{x}$ and $r_{y}$ were fitted by

$$
r_{i}(t) \approx r_{0, i}+\left(\widehat{r}_{i}+v_{r, i} \cdot t+\frac{a_{i} \cdot t^{2}}{2}\right) \sin \left(\frac{2 \pi t}{T_{i}}-\varphi_{i}\right)
$$

assuming a central force $F_{c}$ with an equivalent spring constant $D=-\frac{F_{c}(r)}{r}$ that can be derived from that datafits according to

$$
D_{i}=m \cdot\left(\frac{2 \pi}{T_{i}}\right)^{2}
$$

and a radial acceleration $a_{r}$ stemming from a residual repulsive force $F_{r}=m \cdot a_{r}$. Equation 5 matches the experimental data quite well yielding a coarse description of the acting lateral forces, cf. Fig. 6 (b). The equivalent spring constant $D$ scatters mainly between $3 \mathrm{mN} / \mathrm{mm}$ and $6 \mathrm{mN} / \mathrm{mm}$. For $D>4 \mathrm{mN} / \mathrm{mm}$, the residual repulsive force was in almost every case $<15 \mathrm{mN}$. With some flights where $D<4 \mathrm{mN} / \mathrm{mm}$, however, $F_{r}$ rises up to $\approx 75 \mathrm{mN}$ causing a quickly outwards evolving spiralform in the ground trajectory. In general, $D$ seems to be strongly dependent on initial parameters at launch.

\section{Angular motion}

During the hovering phase, the lightcraft's inclination against the beam propagation axis amounted in average $3.5 \pm 1.9^{\circ}$ (dataset A) to $6.1 \pm 2.2^{\circ}(\mathrm{C})$, together with angular velocity of $31 \pm 14^{\circ} / s(\mathrm{C})$ to $51 \pm 24^{\circ} / s(\mathrm{~A})$. The trajectories of the projected inclination angle $\vartheta_{x}$ and $\vartheta_{y}$, resp., show a spiralform shape as well, cf. Fig. 7 (a). They remind of a gyro's precession in the gravitational field, however, the lightcraft does not spin. The temporal courses were fitted by

$$
\vartheta_{i}(t) \approx \vartheta_{0, i}+\left(\widehat{\vartheta}_{i}+\omega_{i} \cdot t+\frac{\alpha_{i} \cdot t^{2}}{2}\right) \sin \left(\frac{2 \pi t}{T_{\vartheta, i}}-\varphi_{\vartheta, i}\right)
$$

In analogy to the previous section, we assume a backdriving momentum $M_{c}$ with an equivalent torsion spring constant $D_{r}=-\frac{M_{c}(\vartheta)}{\vartheta}$ according to

$$
D_{r, i}=J \cdot\left(\frac{2 \pi}{T_{\vartheta, i}}\right)^{2}
$$

and an angular acceleration $\alpha_{r}$ with a corresponding repulsive momentum $M_{\vartheta}=J \cdot \alpha$. The equivalent torsion spring constant $D_{r}$ is mostly in the range of $\approx 0.1$ to $0.4 \mathrm{mNm} /{ }^{\circ}$ and the residual repulsive momentum $M_{\vartheta}$ varies from $\approx 0.1$ to $0.3 \mathrm{mNm}$, cf. Fig. 7 (b). Higher values of $M_{\vartheta}$ are found for very low $D_{r}$ and surprisingly for very high values of $D_{r}$ which remains unexplained. 


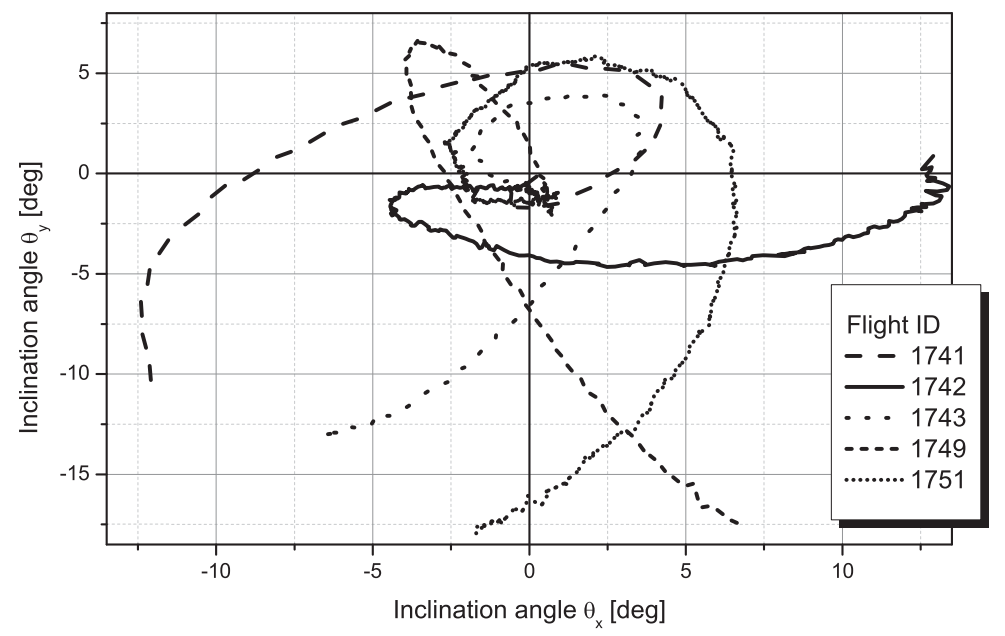

(a)

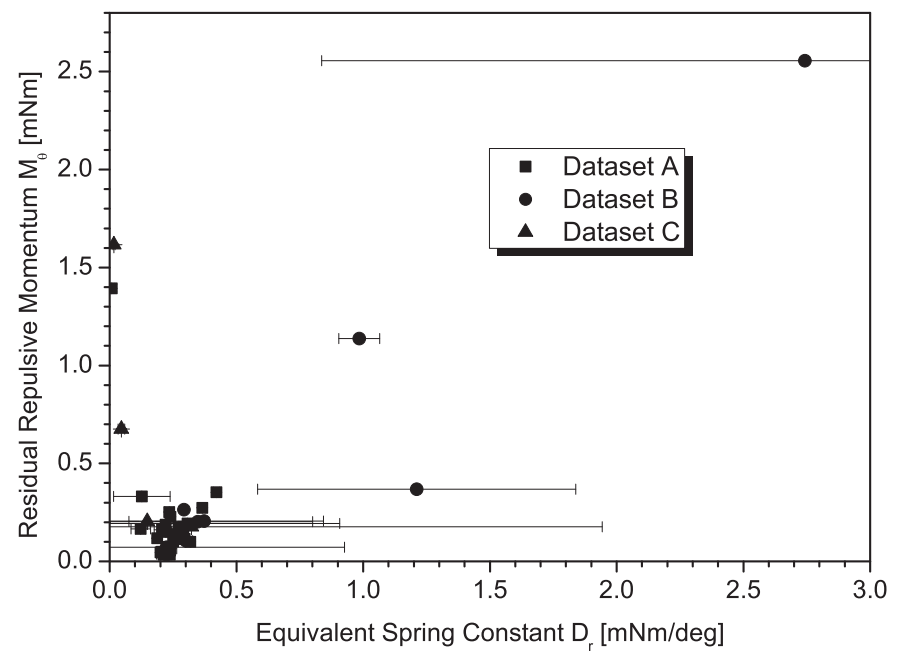

(b)

FIGURE 7. Angular motion: The spiralform trajectories (a) of the projections of the inclination angle $\vartheta$ into the $x-z$ and $y-z$ plane, resp., can be deduced from (b) an equivalent spring constant $D_{r}$ and a residual repulsive momentum $M_{\vartheta}$.

\section{Coupling of lateral and angular motion}

For each plane of projection, lateral and angular motion are linked by a characteristic phase shift $\Delta \varphi_{i}=\varphi_{\vartheta, i}-\varphi_{i}$ yielding a spiralform $r_{i}-\vartheta_{i}$ graph exhibiting a counterclockwise motion, cf. Fig. 8. For any attitude, the lightcraft first directs the apex towards the beam center (if directed outwards) and then moves laterally back to it. Having 


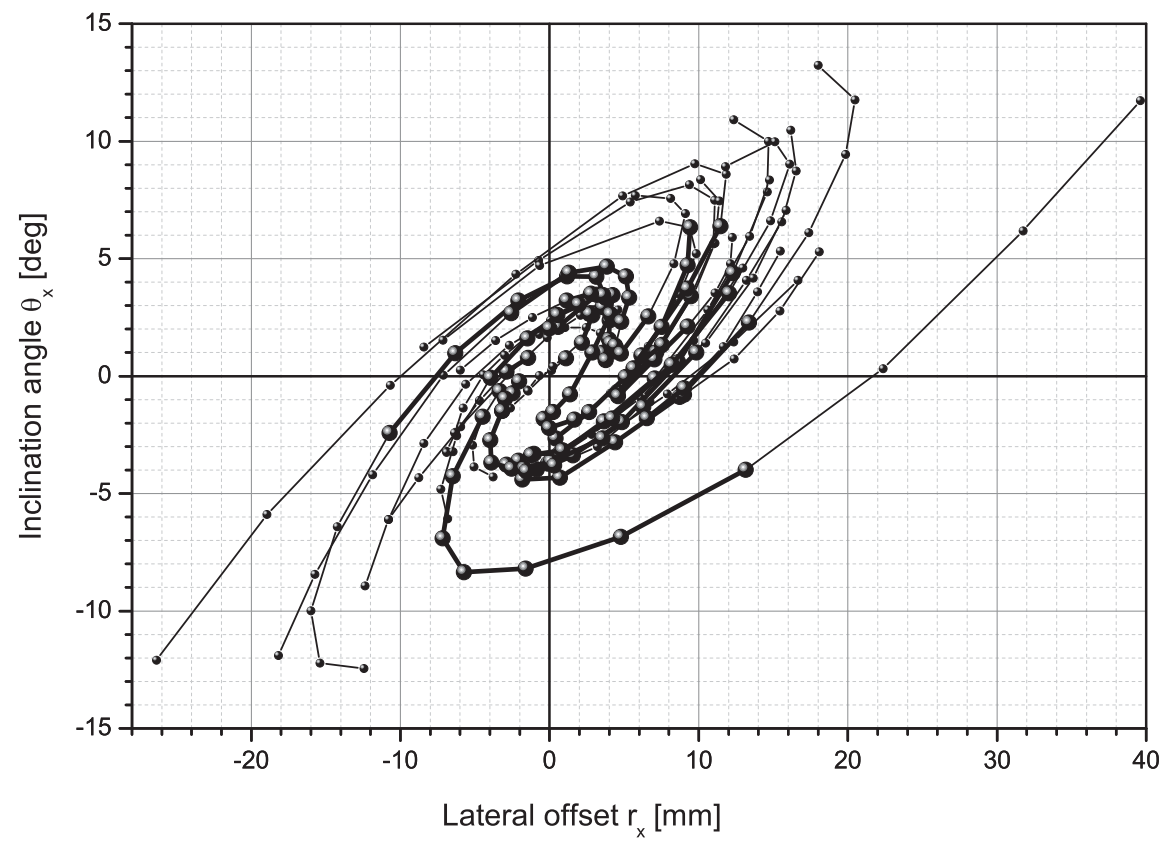

FIGURE 8. Interdependency of inclination angle $\vartheta_{x}$ and $r_{x}$ for the 31 flights of dataset A, X-z plane of projection. The large dots indicate the lightcraft's attitude instantaneously before a laser pulse during the hovering period. The laser pulses during descent and fall are represented by the small dots.

crossed the beam-center, $\vartheta$ is re-orientated first, followed by back-driving momentum towards the beam center again. This shows that, as already noted in [7], lateral and angular motion are strongly connected with each other. From the datafits of the previous section, $\Delta \varphi_{x}=34 \pm 123^{\circ}$ and $\Delta \varphi_{y}=22 \pm 110^{\circ}$ can be derived as an average over all datasets. Though the flight trajectories themselves seem to be quite unpredictable, this characteristic motion has been found for all datasets.

Hence, the large scattering of $D$ and $D_{r}$ might partly be attributed to the dependency on $\vartheta$ and $r$ that was neglected in the corresponding datafits. Therefore, lateral and angular momentum coupling coefficients were modeled with respect to both $\vartheta$ and $r$. The datafits of a linearization by

$$
c_{r} \cdot r+c_{\vartheta} \cdot \vartheta
$$

are shown in Tab. 3 and Tab. 4. Inserting $P_{a v}$, the equivalent spring constants were derived as well. For the lateral motion, the influence of the inclination angle on the back-driving momentum is much more pronounced than the one of the lateral offset itself which in some cases (dataset A) causes a repulsive momentum. Therefore, $D^{c}$ is defined indicating the coupling between lateral momentum and angular inclination according to $D^{c}=-c_{\vartheta} \cdot P_{a v}$ and vice versa for the angular motion.

However, a linearization only holds for small deviations from perfect alignment of the craft. With increasing offset and / or tilt, the impact of the laser pulse diminishes since less energy enters the lightcraft at all. Thus, we compared the experimental data 
TABLE 3. Fitting results for lateral momentum coupling by linearization, cf. Eq. 9. The subscript of the dataset refers to the corresponding plane of projection. $N_{p}$ is the number of datapoints.

\begin{tabular}{lrrrrrr}
\hline & & & ${ }^{c_{r}}$ & ${ }^{c_{\vartheta}}$ & $D$ & $D^{c}$ \\
Dataset & $N_{p}$ & $\bar{R}^{2}$ & $\frac{N}{M W \cdot m m}$ & $\frac{N}{M W \cdot d e g}$ & $\frac{m N}{m m}$ & $\frac{m N}{d e g}$ \\
\hline$A_{x-z}$ & 383 & 0.86 & $0.323 \pm 0.052$ & $-3.82 \pm 0.10$ & $-0.81 \pm 0.17$ & $9.55 \pm 1.17$ \\
$A_{y-z}$ & 383 & 0.83 & $0.254 \pm 0.051$ & $-3.65 \pm 0.11$ & $-0.63 \pm 0.15$ & $9.12 \pm 1.12$ \\
$B_{x-z}$ & 186 & 0.23 & $-0.487 \pm 0.141$ & $-2.13 \pm 0.26$ & $1.70 \pm 0.53$ & $7.44 \pm 1.25$ \\
$B_{y-z}$ & 186 & 0.59 & $0.042 \pm 0.048$ & $-1.45 \pm 0.07$ & $-0.15 \pm 0.17$ & $5.09 \pm 0.64$ \\
$C_{x-z}$ & 106 & 0.69 & $0.090 \pm 0.049$ & $-2.34 \pm 0.15$ & $-0.26 \pm 0.14$ & $6.77 \pm 0.83$ \\
$C_{y-z}$ & 106 & 0.94 & $-0.134 \pm 0.031$ & $-2.41 \pm 0.07$ & $0.39 \pm 0.10$ & $6.99 \pm 0.75$ \\
\hline
\end{tabular}

TABLE 4. Fitting results for angular momentum coupling by linearization, cf. Eq. 9. The subscript of the dataset refers to the corresponding plane of projection. $N_{p}$ is the number of datapoints.

\begin{tabular}{|c|c|c|c|c|c|c|}
\hline Dataset & $N_{p}$ & $\overline{R^{2}}$ & $\frac{{ }^{N m}{ }^{{ }}{ }_{r}}{M W \cdot m m}$ & $\begin{array}{c}{ }^{c_{\vartheta}} \\
\frac{N m}{M W \cdot d e g}\end{array}$ & 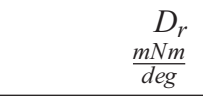 & 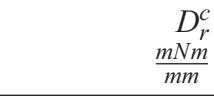 \\
\hline$A_{x-z}$ & 383 & 0.68 & $0.043 \pm 0.002$ & $-0.134 \pm 0.005$ & $0.336 \pm 0.042$ & $-0.109 \pm 0.014$ \\
\hline$A_{y-z}$ & 383 & 0.58 & $0.037 \pm 0.003$ & $-0.125 \pm 0.005$ & $0.311 \pm 0.040$ & $-0.093 \pm 0.013$ \\
\hline$B_{x-z}$ & 186 & 0.01 & $0.008 \pm 0.015$ & $-0.039 \pm 0.028$ & $0.135 \pm 0.099$ & $-0.029 \pm 0.053$ \\
\hline$B_{y-z}$ & 186 & 0.19 & $0.017 \pm 0.003$ & $-0.028 \pm 0.005$ & $0.099 \pm 0.021$ & $-0.061 \pm 0.014$ \\
\hline$C_{x-z}$ & 106 & 0.23 & $0.018 \pm 0.004$ & $-0.052 \pm 0.012$ & $0.152 \pm 0.039$ & $-0.053 \pm 0.013$ \\
\hline$C_{y-z}$ & 106 & 0.38 & $0.010 \pm 0.003$ & $-0.026 \pm 0.006$ & $0.074 \pm 0.020$ & $-0.029 \pm 0.009$ \\
\hline
\end{tabular}

with model results from raytracing that exhibit two pronounced peaks for backdriving momentum and can be approximated by

$$
c_{m, l a t} \approx \frac{c_{\text {lat }}}{\left[1+\left(\frac{r-r_{c}}{\sigma_{r}}\right)^{2}\right]\left[1+\left(\frac{\vartheta-\vartheta_{c}}{\sigma_{\vartheta}}\right)^{2}\right]}-\frac{c_{\text {lat }}}{\left[1+\left(\frac{r+r_{c}}{\sigma_{r}}\right)^{2}\right]\left[1+\left(\frac{\vartheta+\vartheta_{c}}{\sigma_{\vartheta}}\right)^{2}\right]}
$$

The parameters of the corresponding datafits are shown in Tab. 5. In general, the experimental data deviate from the raytracing model with respect to $\operatorname{sign}(\vartheta)$ and their correlation to the model is rather moderate. This can partly be attributed to the database which covers only a small parameter space of $(r, \vartheta)$.

\section{Flight performance model}

The datafits for the components of translational and angular momentum coupling allow for a simulation of the flight performance with given initial parameters. For simplification, we reduce the dimensionality of the problem and assume $\vartheta=\vartheta_{y}, r=r_{y}$ yielding $\vartheta_{x} \equiv 0 \equiv r_{x}$ during the experiment. Inserting the datafit parameters of dataset A and $A_{y-z}$, resp., the hovering duration can be calculated with respect to the lightcraft's initial inclination and offset. This approach is made in analogy to Julia sets with the 
TABLE 5. Fitting results for lateral momentum coupling by a double-peak Lorentz fit, cf. Eq. 10. The subscript of the dataset refers to the corresponding plane of projection. $N_{p}$ is the number of datapoints.

\begin{tabular}{|c|c|c|c|c|c|c|c|}
\hline Dataset & $\begin{array}{c}c_{\text {lat }} \\
\frac{N}{M W}\end{array}$ & $\begin{array}{r}r_{c} \\
m m\end{array}$ & $\begin{array}{r}\sigma_{r} \\
m m\end{array}$ & $\begin{array}{r}\vartheta_{c} \\
d e g\end{array}$ & $\begin{array}{r}\sigma_{\vartheta} \\
d e g\end{array}$ & $N_{p}$ & $\overline{R^{2}}$ \\
\hline$A_{x-z}$ & $47.1 \pm 2.7$ & $1.9 \pm 1.2$ & $30.2 \pm 4.5$ & $-10.7 \pm 0.6$ & $8.3 \pm 1.0$ & 383 & 0.90 \\
\hline$A_{y-z}$ & $64.2 \pm 8.4$ & $-0.9 \pm 0.3$ & $22.3 \pm 1.8$ & $-14.8 \pm 1.8$ & $11.9 \pm 2.4$ & 383 & 0.91 \\
\hline$B_{x-z}$ & $17.6 \pm 4.6$ & $-0.7 \pm 1.2$ & $6.1 \pm 3.5$ & $-5.8 \pm 1.1$ & $2.6 \pm 1.2$ & 186 & 0.18 \\
\hline$B_{y-z}$ & $21.9 \pm 1.7$ & $1.7 \pm 0.7$ & $16.3 \pm 2.7$ & $-10.4 \pm 0.9$ & $8.3 \pm 2.8$ & 186 & 0.65 \\
\hline$C_{x-z}$ & $16 \pm 14$ & $-26 \pm 153$ & $52 \pm 188$ & $-3.3 \pm 0.5$ & $1.1 \pm 0.4$ & 106 & 0.21 \\
\hline$C_{y-z}$ & $27.7 \pm 2.3$ & $-7.0 \pm 6.0$ & $53 \pm 13$ & $-10.1 \pm 1.0$ & $6.6 \pm 1.4$ & 106 & 0.95 \\
\hline Model & $0.78 \pm 0.01^{*}$ & $-21.1 \pm 0.4$ & $38.8 \pm 0.4$ & $0.78 \pm 0.02$ & $2.63 \pm 0.01$ & 8957 & 0.94 \\
\hline
\end{tabular}

* in arbitrary units

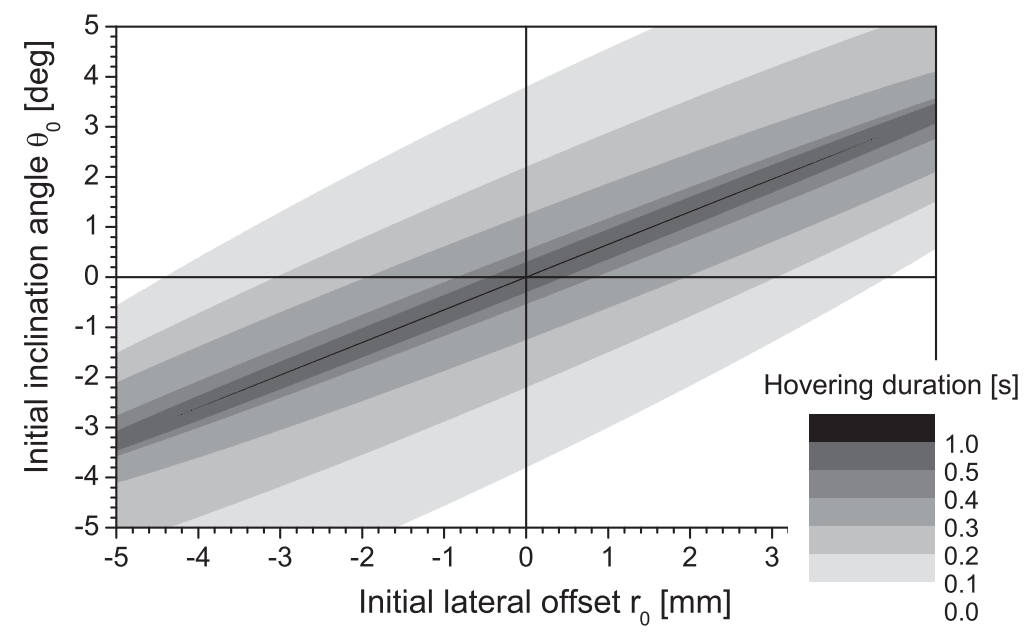

FIGURE 9. Model calculation of the hovering duration vs. initial parameters at launch with fit parameters on momentum coupling for dataset $A_{y-z}$.

launch parameters as a seed value and the laws of motion as the corresponding function on the complex numbers being iterated from pulse to pulse until dropoff from the laser beam. A detailed explanation is given in [8]. It can be seen from Fig. 9 that the calculated hovering time is mainly limited to $\approx 0.5 \mathrm{~s}$. Only for perfect alignment or specific combinations of $r$ and $\vartheta$ longer hovering states up to $\approx 3.25 \mathrm{~s}$ might be achieved theoretically. This is in good accordance to the experimental findings: The lateral alignment accuracy amounted to $\pm 1 \mathrm{~mm}$ in the experiment allowing for great variation in the hovering time, as can be seen from the calculation data. Furthermore, the upper temporal limit of the hovering state can be explained by a mismatch of the repetition period, since $c_{z}=273.9 \mathrm{~N} / \mathrm{MW}$ would demand for $T_{\exp }=43.4 \mathrm{~ms}$ instead of the experimental value of $44 \mathrm{~ms}$. 


\section{Control loops}

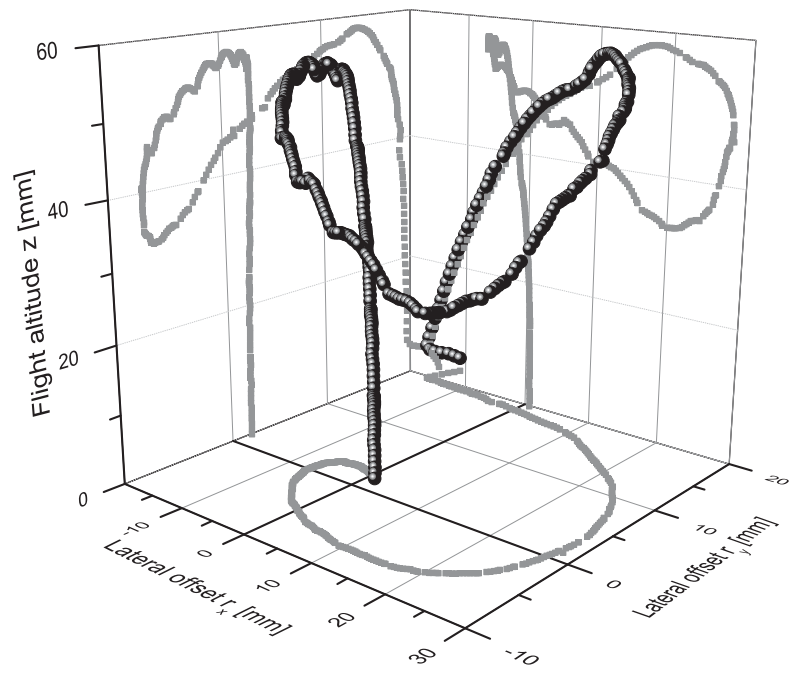

(a)

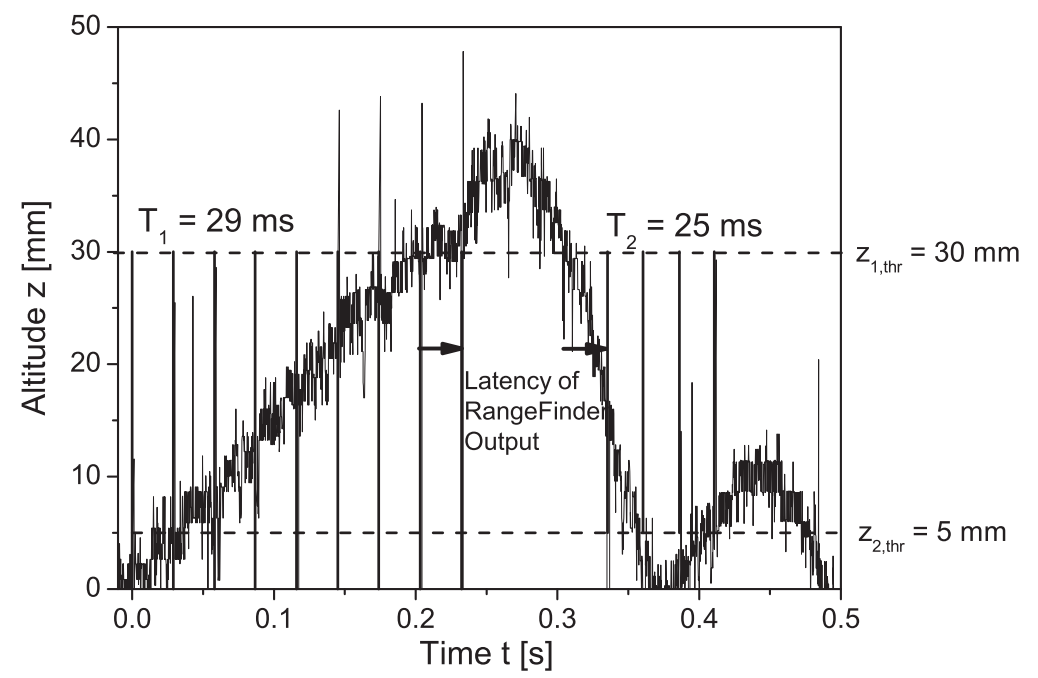

(b)

FIGURE 10. Trajectory (a) of a sample flight, where at the end of the hovering period the laser accidently started to fire in an arc discharge with $\approx 100 \mathrm{~Hz}$ raising the lightcraft again and providing for reorientation the beam center. In a dynamic control loop (b) the laser burst was coupled to a laser rangefinder output to stop when the lightcraft climbs beyond a threshold altitude $z_{1, t h r}$ and for restart with a higher repetition rate if it falls below it again.

By chance, we observed a stabilization of the hovering state because of laser malfunction during the burst, cf. Fig. 10 (a). During the descent of the lightcraft the laser suddenly started firing with a 4 times higher repetition rate. The lightcraft rised and hovered again before it finally dropped down. Since axial momentum coupling decreases with inclination and lateral offset, the hovering time with a predefined burst was limited 
in our experiments. Hence, we took into account for the decrease of $c_{m, z}$ by choosing a higher repetition rate for the hovering sequence and built a dynamic control loop by means of a laser rangefinder AR4000RET by Acuity Research. The range finder pointed down from the top of the flight range to a retroreflective coating that was applied to the lightcraft's protective cap. It operated at a sample rate of $5 \mathrm{kHz}$ with an onboard data averaging and filtering of artifacts that was implemented by a $\mathrm{C}++$ script on the corresponding high speed interface (HSIF) yielding a $200 \mathrm{~Hz}$ data output on two channels indicating whether certain threshold altitudes were exceeded or not. These data were sampled and averaged with an analog input module (\# 9239 by National Instruments) on board the real-time control of the laser supplying status information of the flight.

In the experiment, the laser was fired with a long repetition period $T_{1}$ until the hovering altitude $z_{1, t h r}$ was exceeded. When the lightcraft fell below $z_{1, t h r}$, the laser was turned on again, but with a shorter repetition period $T_{2}$. The lower threshold altitude $z_{2, t h r}$ was used for final laser shutdown. However, these experiments only show a restricted proof of principle since a restabilization of the hovering state failed. This was mainly due to the large latency $\approx 30 \mathrm{~ms}$ of the rangefinder output which was layouted as a variable power supply for scanning motors. After changes on the on the HSIF circuit board, both range finder and $\mathrm{CO}_{2}$ laser showed malfuncions and the experiments had to be aborted in favor of maintainance work.

Moreover, an alternative to a remote (laser-based) control loop was already presented in $[6,11]$. By tilting the ignition pin (or an ablative propellant rod) against the symmetry axis of the nozzle, $c_{m, l a t}$ and $c_{L}$ can be significantly varied allowing for an onboard control loop. Experimental data on steering performance with respect to lightcraft attitude are subject of ongoing analysis.

\section{CONCLUSIONS}

In order to keep the technical effort and structural mass of a prospective lightcraft mission as low as possible we examined the inherent stabilization properties of the pulsed laser propulsion process in a parabolic thruster with respect to axial, lateral and angular momentum in a hovering experiment. Though our intention was to establish a dynamically stable hovering state for several seconds, we were only able to achieve a hovering duration of max. $\approx 0.36 s$, independent of the repetition rate.

Impulse compenents were characterized with respect to the lightcraft's attitude showing a pronounced decrease with offset and inclination shortening the hovering time significantly. Moreover, backdriving lateral and angular momentum components were found exhibiting non-linear characteristics and a strong coupling that causes spiralform trajectories in the $r-\vartheta$ plane. However, these components are not sufficient to grant for a stabilized oscillation around the beam center which might be attributed to a twist angle between the momentary plane of the lateral offset and the plane of inclination. Nevertheless, it should be pointed out that the laser burst was optimized for the compensation of the gravitational force and might be optimized at a different repetition rate for adjustment of backdriving momentum components. These issues can be modeled with a Julia set approach based on the fit parameters of momentum coupling. The experimental data are in moderate accordance with model data from raytracing. This might be improved by 
an extended 3D model taking into account for the twist angle in both raytracing model and momentum coupling data.

\section{ACKNOWLEDGMENTS}

The authors want to thank Sebastian Walther for technical support in the laboratory and laser operation, Uli Nesper, Frank Schramm and Milan Hodak for support from the DLR jobshop, Metalldrückerei Klose, Pinneberg for nozzle manufacturing and 4D concepts, Gross-Gerau, for fabrication of the protective lightcraft caps. A special thank is given to Stephanie Karg and David Sperber for fruitful discussions.

\section{REFERENCES}

1. L. Myrabo, World record flights of beam-riding rocket lightcraft: Demonstration of disruptive propulsion technology, AIAA paper 2001-3798 (2001).

2. M. Libeau, L. Myrabo, M. Filippelli, and J. McInerney, "Combined Theoretical and Experimental Flight Dynamics Investigation of a Laser-Propelled Vehicle," in $1^{\text {st }}$ International Symposium on Beamed Energy Propulsion, edited by A. Pakhomov, AIP Conference Proceedings 664, American Institute of Physics, Melville, New York, 2003, pp. 125-137.

3. M. Libeau, and L. Myrabo, "Off-Axis and Angular Impulse Measurements on a Lightcraft Engine," in $3^{\text {rd }}$ International Symposium on Beamed Energy Propulsion, edited by A. Pakhomov, and L. Myrabo, AIP Conference Proceedings 766, American Institute of Physics, Melville, New York, 2005, pp. 166-177.

4. D. Kenoyer, K. Anderson, and L. Myrabo, "Validation and Calibration of a 6-DOF Laser Propelled Lightcraft Flight Dynamics Model vs. Experimental Data," in $5^{\text {th }}$ International Symposium on Beamed Energy Propulsion, edited by C. Phipps, K. Komurasaki, and J. Sinko, AIP Conference Proceedings 997, American Institute of Physics, Melville, New York, 2010, pp. 325-337.

5. D. Kenoyer, L. Myrabo, S. Notaro, and P. Bragulla, "Experimental Investigation into Beam-Riding Physics of Lightcraft Engines: Progress Report," in $6^{\text {th }}$ International Symposium on Beamed Energy Propulsion, edited by A. Pakhomov, AIP Conference Proceedings 1230, American Institute of Physics, Melville, New York, 2010, pp. 17-29.

6. S. Scharring, D. Hoffmann, H.-A. Eckel, and H.-P. Röser, Acta Astronautica 65, 1599-1615 (2009).

7. S. Scharring, H.-A. Eckel, and H.-P. Röser, "High speed analysis of free flights with a parabolic thruster," in $6^{\text {th }}$ International Symposium on Beamed Energy Propulsion, edited by C. Phipps, K. Komurasaki, and J. Sinko, AIP Conference Proceedings 1230, American Institute of Physics, Melville, New York, 2010, pp. 77-88.

8. S. Scharring, H.-A. Eckel, and H.-P. Röser, International Journal of Aerospace Innovations 3, 15-31 (2011).

9. Lasers and laser-related equipment - test methods for laser beam widths, divergence angles and beam propagation ratios - part i: Stigmatic and simple astigmatic beams, Tech. Rep. ISO 11146-1:2005(E), ISO copyright office, Geneva, Switzerland (2005).

10. W. Mayerhofer, T. Hall, W. Riede, E. Zeyfang, and S. Walther, Repetitively-pulsed multigas high power laser, Internal Report IB 44102/99, DLR - Institute of Technical Physics (1999), (in German).

11. S. Scharring, D. Hoffmann, H.-A. Eckel, and H.-P. Röser, "Remotely controlled Steering Gear for a Laser-Driven Rocket with a Parabolic Thruster," in $6^{\text {th }}$ International Symposium on Beamed Energy Propulsion, edited by C. Phipps, K. Komurasaki, and J. Sinko, AIP Conference Proceedings 1230, American Institute of Physics, Melville, New York, 2010, pp. 89-100. 